\title{
REGULARITY OF THE CATEGORY OF KELLEY SPACES
}

\author{
F. Cagliari, S. Mantovani and E.M. Vitale
}

\begin{abstract}
We show that the cartesian closed category of compactly generated Hausdorff spaces is regular, but is neither exact, nor locally cartesian closed. In fact we find a coequalizer of an equivalence relation which is not stable under pullbacks.
\end{abstract}

\section{Introduction}

It is well known that the category Top of topological spaces has not a good behaviour with respect to two fundamental constructions, namely function spaces and coequalizers of equivalence relations, since Top is neither cartesian closed, nor exact, nor regular (by exact category we mean a regular category with effective equivalence relations, cf.[B]). (Day and Kelly in [DK] characterized both cartesian objects and universal quotients of Top).

In order to avoid these difficulties which arise in Top, one can try to find appropriate categories of topological spaces which do have such properties.

From Manes theorem, we know that the category Hcompt of compact Hausdorff spaces is exact, but it is not cartesian closed (see the characterization of exponential objects and maps in Hcompt in $[\mathrm{CM}]$ ). We can enlarge this category in order to obtain cartesian closedness: it is sufficient to consider the category Kelley of Kelley spaces (compactly generated Hausdorff spaces). (cf. [S],[GZ]) The problem now is to see if the property of exactness still remains or at least to see whether some weaker proerty holds.

The first result of this paper is to show that Kelley is regular.

Since a necessary condition for exactness is that coequalizers of equivalence relations are pullback stable, in order to know if Kelley is exact, we could investigate the behaviour of pullback functors with respect to the preservation of coequalizers. Via the special adjoint functor theorem, in this case the preservation of coequalizers and coproducts is equivalent to the existence of a right adjoint to pullback functors, that is to the local cartesian closedness. Furthermore in Kelley coproducts coincide with topological sums and we will easily show that they are stable under pullback, so that preservation of coequalizers would tell us that Kelley is

Key words and phrases. Compactly generated spaces, regular and exact categories,locally cartesian closed categories.

F. Cagliari, Dipartimento di Matematica, Piazza di Porta S.Donato, 5- 40126 Bologna - Italy

S. Mantovani, Dipartimento di Matematica, Corso Duca Abruzzi, 24- 10129 Torino- Italy

E.M. Vitale, Institut de Mathématique Pure et Appliquée, Université Catholique de Louvain, 1348 Louvain-la-Neuve, Belgique 
also locally cartesian closed. But this is not the case. In fact what we find is that in this category pullback functors preserve regular epimorphisms, but not coequalizers, not even coequalizers of equivalence relations, which means that Kelley is also not exact. (Notice that, since in the case of Hcompt pullback functors preserve coequalizers, but fail to preserves coproducts, both the categories we are interested in are not locally cartesian closed, but for opposite reasons.)

As a final remark, we can look at the exact completion, say $\mathcal{E}_{e x / r e g}$, of Kelley, which exists because Kelley is regular (cf.[CW]). Since in Kelley coproducts are disjoint and universal, the same happens in $\mathcal{E}_{\text {ex/reg }}$. This means that the only thing missing to $\mathcal{E}_{e x / r e g}$ for being a Grothendieck topos is, according to the Giraud's conditions (cf., for example [BW] p.238), a set of regular generators. But this is untrue, as Johnstone shows in [J] 10.8 and 10.9, since the existence of such a set in $\mathcal{E}_{\text {ex } / \text { reg }}$ would imply the existence of a set of regular generators for Kelley.

We would like to thank A. Carboni for many helpful conversations and suggestions.

Let us start with the first problem faced in the introduction, namely the regularity of Kelley.

Definition. (cf. $[\mathrm{B}],[\mathrm{L}])$ We recall that a category $\mathcal{C}$ is said to be regular if the following holds:

1) $\mathcal{C}$ has finite limits

2) Every morphism has the (regular epimorphism, monomorphism)-factorization

3) Regular epimorphisms are stable under pullbacks, that is a pullback of a regular epimorphism along any morphism is a regular epimorphism.

$\mathcal{C}$ is said to be exact when it is regular and has effective equivalence relations (which means that they coincide with the kernel pair of their coequalizer).

The first two conditions are satisfied in any coreflective subcategory (the inclusion functor has a right adjoint) of the category Haus of Hausdorff spaces. For the last condition, we need some additional hypothesis, as the following shows:

Lemma. Let $\mathcal{C}$ be a cartesian closed category with regular epimorphisms stable under pullbacks along regular monomorphisms.

Then regular epimorphisms are stable under pullbacks along any morphism.

Proof. Let $q: X \rightarrow Y$ be a regular epimorphism in $\mathcal{C}$ and consider its pullback $q^{\prime}$ along any morphism $f: T \rightarrow Y$

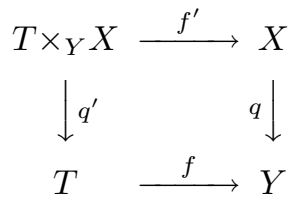


As a consequence the following diagram is again a pullback diagram:

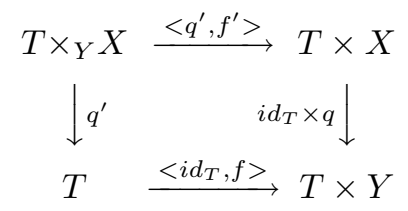

$\left(i d_{T} \times q\right): T \times X \rightarrow T \times Y$ is a regular epimorphism, since $T \times(-)$ preserves colimits ( $\mathcal{C}$ is cartesian closed). Furthermore, if we denote by $p_{T}, p_{Y}$ the projections of $T \times Y$ onto its factors, $\left\langle i d_{T}, f>: T \rightarrow T \times Y\right.$ is the equalizer of $\left(f p_{T}, p_{Y}\right)$. Then $q^{\prime}$ is obtained as the pullback of a regular epimorphism along a regular monomorphism, so it is itself a regular epimorphism.

Applying the previous Lemma to the category of Kelley spaces we obtain (cf. $[\mathrm{V}])$ :

Theorem. The category of Kelley spaces is regular.

Proof. . Since Kelley is coreflective (and then bicoreflective) in the category of Hausdorff spaces, it is closed under quotients in Haus and the regular epimorphisms in Kelley are exactly the topological quotients. Thus Kelley has the (regular epimorphism=quotient, monomorphism)-factorization. Furthermore, Kelley is closed under the formation of equalizers in Haus and then regular monomorphisms coincide with closed embeddings. Since topological quotients are stable under pullbacks along closed embeddings, we can apply the previous lemma, showing that Kelley is regular.

Remark. The previous theorem holds in any cartesian closed category of topological spaces having the (quotient,injection)-factorization and equalizers which are closed embeddings. In particular we have the regularity of the category of weakly Hausdorff k-spaces ( cf. e.g [LG]) and of the category of sequential Hausdorff spaces $(\operatorname{cf}[\mathrm{J}])$.

Now we want to investigate the behaviour of pullback functors related to sums and coequalizers. In the case of Hcompt, where coequalizers are preserved by pullbacks along any morphism, coproducts are not preserved. In fact, if you take the Stone - $\check{C}$ ech compactification of the natural numbers $\beta \mathrm{N}=\coprod_{1}^{+\infty}\{n\}$, the pullbacks of the inclusions $i_{n}:\{n\} \rightarrow \beta \mathrm{N}$, along the inclusion of $\beta \mathrm{N} \backslash \mathrm{N}$ in $\beta \mathrm{N}$ are the inclusions of the empty set into $\beta \mathrm{N} \backslash \mathrm{N}$, which clearly does not coincide with the coproduct of countable copies of the empty set.

In the case of Kelley, coproducts coincide with topological sums and are preserved by pullbacks along any morphism. In fact, consider a coproduct $\sum_{I} X_{i}$ in Kelley: since it is a topological sum, its pullback $P$ in Haus (which is the same as in Top) is the topological sum of the pullbacks $X_{i}^{\prime}$ in Haus of any cofactor. But the pullback $c P$ in Kelley of $\sum_{I} X_{i}$ is the coreflection of $P=\sum_{I} X_{i}^{\prime}$ in Kelley, and, since this coreflection preserves topological sums, $c P$ coincides with the coproduct $\sum_{I} c X_{i}^{\prime}$ of the coreflection $c X_{i}^{\prime}$ of any cofactor $X_{i}^{\prime}$. But any of these $c X_{i}^{\prime}$ is exactly the pullback of $X_{i}$ in Kelley and then we have that coproducts are universal also in Kelley. 
The reason which does not allow Kelley to be locally cartesian closed lays in the subtle, but substantial difference between preservation along pullbacks of regular epimorphisms and preservation of coequalizers. In particular we will find an equivalence relation whose coequalizer is not preserved. This example shows at the same time that Kelley can be neither locally cartesian closed, nor exact, since in exact categories equivalence relations are effective and therefore their coequalizers must be stable under pullbacks.

Proposition. The category of Kelley spaces is neither locally cartesian closed nor exact.

Proof. Consider the unit interval $I=[0,1]$ and the coproduct $I+I$ of two copies of $I$ with the two coprojections $i_{1}, i_{2}: I \rightarrow I+I$. Denote by $I_{1}$ and by $I_{2}$ the copies of $I$ in $I+I$ given respectively by $i_{1}(I)$ and $i_{2}(I)$. Then the product $(I+I) \times(I+I)$ can be identified with $\left(I_{1}\right)^{2}+\left(I_{2}\right)^{2}+I_{1} \times I_{2}+I_{2} \times I_{1}$. Let $\Delta_{1}, \Delta_{2}, \Delta_{1,2}$ and $\Delta_{2,1}$ be the diagonals of any of the squares, in the same order. Consider now the subspace $R$ of $(I+I) \times(I+I)$ given (with suitable identifications) by $\Delta_{1}+\Delta_{2}+$ $\Delta_{1,2} \backslash\{(0,0)\}+\Delta_{2,1} \backslash\{(0,0)\} . R$ is not compact, but it is still a Kelley space, since it is first countable. Furthermore $R$ is an equivalence relation on $I+I$, namely the relation whose elements are the pairs $\left(i_{j}(0), i_{j}(0)\right)$ for $j=1,2$ and $\left(i_{k}(x), i_{h}(x),\right)$, for $k, h=1,2$ and $x \in I, x \neq 0$. Roughly speaking, any element of $I_{1}$ but 0 is in relation with the same element in the other copy $I_{2}$ and any of the copies of 0 is in relation only with itself. The coequalizer of this equivalence relation in Kelley is the same as the coequalizer $q$ in Haus (Kelley is coreflective in Haus). On the other hand, $q$ is obtained taking the reflection in Haus of the coequalizer $\bar{q}$ in Top. It easy to see that $\bar{q}: I+I \rightarrow J$ identifies $\left.I_{1} \backslash\{0\}\right)$ with $I_{2} \backslash\{0\}$ ), while $\bar{q}$ distinguishes the two copies of 0 in $I+I$. Furthermore these two points have not disjoint neighbourhoods in $J$, which means that $J$ is not Hausdorff. So the Hausdorff reflection of $J$ is homeomorphic to $I$.

Consider now the inclusion $m:\{0\} \rightarrow I$ of the point 0 in $I$. We want to show that the pullback along $m$ does not preserve the coequalizer $q$.

The pullback $q^{\prime}$ of $q$ along $m$ is the quotient of the two-point discrete space $D_{2}$ over the singleton:

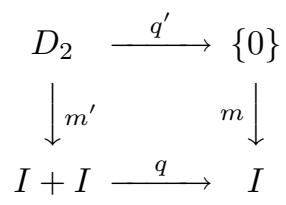

If coequalizers were preserved by pullbacks, $q^{\prime}$ should be the coequalizer of the pair given by the pullback of $q r_{0}$ and $q r_{1}$ along $m$, where $r_{0}, r_{1}$ are the two projections of $R$ on $I+I$. This pair is given by $(i d, i d)$, where $i d$ is the identity of $D_{2}$. But the coequalizer of $(i d, i d)$ must be an isomorphism and so cannot coincide with $q^{\prime}$.

\section{REFERENCES}

[B] M.Barr, Exact Categories, Exact Categories and Categories of Sheaves, LNM 236, Springer - Verlag, New York Heidelberg Berlin, 1985.

[BW] M.Barr and C.Wells, Toposes, Triples and Theories, Springer- Verlag, New York Heidelberg Berlin, 1971. 
[CW] A.Carboni and R.F.C.Walters, Cartesian Bicategories I, Jour. Pure and Appl. Algebra $\underline{45}$ (1987), 11-32.

[CM] F.Cagliari and S.Mantovani, Local homeomorphisms as the exponentiable morphisms in Compact Hausdorff spaces, Topology and its Appl. $\underline{41}$ (1991), 263-272.

[DK] B.J.Day and G.M.Kelly, On topological quotient maps preserved by pullbacks or products, Proc. Camb. Phil. Soc. 67 (1970), 555-558.

[GZ] P.Gabriel and M.Zisman, Categories of Fractions and Homotopy Theory, Springer-Verlag, New York Heidelberg Berlin, 1967.

[J] P.T.Jonhstone, On a topological topos, Proc. London Math. Soc. 38 (1979), 237-271.

[L] F.W.Lawvere, Theory of Categories over a Base Topos, Ist. Mat. Univ. Perugia, 1972-73.

[LG] L.G.Lewis, Open maps, colinits and a convenient category of fibred spaces, Top. and Appl. 19 (1985), 75-89.

[S] N.E.Steenrod, A convenient category of topological spaces, Michigan Math. J. 14 (1967), 133-152.

[V] E.M.Vitale, Left covering functors, Ph. D. thesis, Louvain-la-Neuve, 1994. 